\title{
Erratum to: Viral load of EBV DNAemia is a predictor of EBV-related post-transplant lymphoproliferative disorders in pediatric renal transplant recipients
}

\author{
Elisa Colombini ${ }^{1}$ - Isabella Guzzo ${ }^{1}$ - Federica Morolli ${ }^{1}$ - Germana Longo ${ }^{2}$ - Cristina Russo ${ }^{3}$. \\ Alessandra Lombardi ${ }^{4}$ Pietro Merli ${ }^{4} \cdot$ Luisa Barzon $^{5} \cdot$ Luisa Murer $^{2} \cdot$ Simone Piga $^{6}$. \\ Marta Luisa Ciofi degli Atti ${ }^{6}$. Franco Locatelli ${ }^{4,7} \cdot$ Luca Dello Strologo $^{1}$
}

Published online: 10 July 2017

(C) IPNA 2017

\section{Erratum to: Pediatr Nephrol}

DOI: 10.1007/s00467-017-3627-2

Throughout this article the authors erroneously reported EBV DNA quantities in copies per microliter $(\mu \mathrm{l})$, rather than copies per milliliter (ml). The numbers are correct throughout, but are given with the wrong unit of volume.

The authors sincerely apologize for their mistake and for any inconvenience caused.

Luca Dello Strologo

luca.dellostrologo@opbg.net

Elisa Colombini

elisa.colombini@inwind.it

Isabella Guzzo

isabella.guzzo@opbg.net

Federica Morolli

federica.morolli@opbg.net

Germana Longo

longo.germana@libero.it

Cristina Russo

cristina.russo@opbg.net

Alessandra Lombardi

alessandra.lombardi@opbg.net

Pietro Merli

pietro.merli@opbg.net

Luisa Barzon

luisa.barzon@unipd.it
Luisa Murer

murer@pediatria.unipd.it

Simone Piga

simone.piga@opbg.net

Marta Luisa Ciofi degli Atti marta.ciofidegliatti@opbg.net

1 Pediatric Nephrology and Renal Transplant Unit, Bambino Gesù Children's Hospital-IRCCS, Piazza S. Onofrio 4, 00165 Rome, Italy

2 Pediatric Nephrology, Dialysis and Transplantation Unit, Department of Pediatrics, University of Padova, Padova, Italy

3 Virology Unit, Bambino Gesù Children's Research Hospital-IRCCS, Rome, Italy

4 Hematology and Oncology, Bambino Gesù Children's Research Hospital - RCCS, Rome, Italy

5 Department of Molecular Medicine, University of Padua, Padua, Italy

6 Unit of Clinical Epidemiology, Bambino Gesù Children's Research Hospital-IRCCS, Rome, Italy

7 University of Pavia, Pavia, Italy 